\title{
Stability Analysis of Symmetrical Rotors Partially Filled with a Viscous Incompressible Fluid
}

\author{
ZHU CHANGSHENG* \\ Department of Electrical Engineering, Zhejiang University, Hangzhou, 310027, Zhejiang, P.R. of China
}

(Received in final form 21 June 2000)

\begin{abstract}
On the basis of the linearized fluid forces acting on the rotor obtained directly by using the two-dimensional Navier-Stokes equations, the stability of symmetrical rotors with a cylindrical chamber partially filled with a viscous incompressible fluid is investigated in this paper. The effects of the parameters of rotor system, such as external damping ratio, fluid fill ratio, Reynolds number and mass ratio, on the unstable regions are analyzed. It is shown that for the stability analysis of fluid filled rotor systems with external damping, the effect of the fluid viscosity on the stability should be considered. When the fluid viscosity is included, the adding external damping will make the system more stable and two unstable regions may exist even if rotors are isotropic in some cases.
\end{abstract}

Keywords: Rotordynamics; Instability; Rotating fluid; $\mathrm{N}-\mathrm{S}$ equation; Rotors

\section{INTRODUCTION}

For the rotating machinery with cavity components, such as fluid-filled centrifuges and separators, fluid-cooled gas turbines, spin-stabilized satellites as well as rockets containing liquid fuels, there is an amount of fluid trapped in the cavity when the rotor rotates. The interaction between the rotating rotor and the enclosed fluid may lead to self-excited vibration or instability in a certain region of rotational speeds. The instability of rotors partially filled with fluid was first observed by Kollmann (1962), and then theoretically explained by many researchers. The theoretical models used hitherto are basically divided into two groups: the in-viscous fluid and the viscous fluid. In the in-viscous fluid model, one of the most important conclusions is that adding external damping on the rotor system will cause the rotor system to become unstable at any speeds. This is not in agreement with the facts that the unstable motions observed in experiments only occur in a certain range of rotational speeds and that there is a certain amount of external damping in every

*Present address: Center of Vibration Engineering, Mechanical Engineering Department, Imperial College, Exhibition Road, London SW7 2BX, UK. e-mail: c.s.zhu@ic.ac.uk, cszhu@hotmail.com 
experimental rig. The reason for this is that the effect of the fluid viscosity is not considered. The unstable region derived from the viscous fluid model is finite, but the conclusions about the effect of external damping on the rotor stability obtained by using different solving methods do not agree with each other. For example, Hendricks and Morton (1979) showed that the external damping makes the rotor system become more unstable; but Saito and Someya (1980); Holm and Träger (1991) showed that the external damping makes the system more stable. Therefore, there are lots of problems to be studied in the stability of the rotors partially filled with viscous fluid.

On the basis of the linearized fluid forces acting on the rotor in the two-dimensional case obtained directly by using the Navier - Stokes equations, the objectives of this paper are to investigate the stability of symmetrical rotor systems partially filled with the viscous incompressible fluid and to analyze the effects of the system parameters, such as the external damping ratio, fluid fill ratio, Reynolds number at the critical speed and mass ratio, on the stability of the fluid filled rotor system.

\section{ROTATING VISCOUS FLUID DYNAMICS}

Basically, the motion of the rotor is coupled with the fluid motion in the chamber. During the steady state operation, the rotor will be exposed to a deflection, and waves will be excited in the rotating fluid layer. These waves will produce fluid forces on the rotor which depends on the unknown whirling speed of the rotor, rotational speed, structure of the chamber and properties of the fluid. The fluid forces are incorporated into the equations of motion of the rotor, to give the conditions which must be satisfied if the instability occurs. Therefore, the key problem in analyzing the dynamics of the rotor partially filled with fluid is to obtain the fluid forces acting on the rotor.

The following assumptions are made in order to analyze the linearized fluid forces acting on the rotor. (1) The fluid chamber is an axis-symmetrical rigid cylinder and is totally balance, the properties of the fluid, such as fluid kinematic viscosity and density are constants; (2) The fluid is viscous incompressible and the surface tension effects are negligible. During operation, no fluid enters or leaves the chamber; (3) The effect of the gravity force is neglected in comparison with the centrifugal force; (4) The motion of the fluid in the chamber is assumed to be uniform in the axial direction and independent of axial position; (5) The rotor is driven at a constant rotational speed $\Omega$. Under the above assumptions, the equations of motion of the fluid in the polar co-ordinate system rotating at the speed $\Omega$, can be written from the two-dimensional Navier-Stokes equations as follows:

$$
\begin{aligned}
\frac{\partial u}{\partial t}+ & u \frac{\partial u}{\partial r}+\frac{u}{r} \frac{\partial u}{\partial \phi}-\frac{v^{2}}{r}-r \Omega^{2}-2 v \Omega \\
= & -\frac{1}{\rho} \frac{\partial p}{\partial r}+\mu\left(\Delta u-\frac{2}{r^{2}} \frac{\partial v}{\partial \phi}-\frac{u}{r^{2}}\right) \\
& +a \varepsilon \omega^{2} \exp [i(\omega-\Omega) t-i \phi] \\
\frac{\partial v}{\partial t}+ & u \frac{\partial v}{\partial r}+\frac{v}{r} \frac{\partial v}{\partial \phi}+\frac{v u}{r}+2 v \Omega \\
= & -\frac{1}{\rho r} \frac{\partial p}{\partial \phi}+\mu\left(\Delta v+\frac{2}{r^{2}} \frac{\partial u}{\partial \phi}-\frac{v}{r^{2}}\right) \\
& -a \varepsilon \omega^{2} i \exp [i(\omega-\Omega) t-i \phi]
\end{aligned}
$$

where $\Delta$ is the Laplacian operator, $\mu$ and $\rho$ are the kinematic viscosity and the density of fluid. $u$ and $v$ are the velocities in the $r$ and $\phi$-directions, respectively. $\omega$ is the whirling speed of the rotor, $\varepsilon$ is the eccentricity ratio of the chamber motion relative to the inner radius of the chamber $a, p$ is the fluid pressure, and $a$ is the inner radius of the chamber. $i=\sqrt{-1}$.

The equation of continuity for the incompressible rotating fluid is given by

$$
\frac{\partial(r u)}{\partial r}+\frac{\partial v}{\partial \phi}=0
$$


The boundary condition at the wetted rotor $\operatorname{surface}(r=a)$ is

$$
u(a)=0
$$

During the rotor's whirling, the position of the free surface of the fluid is defined by $R_{b}=b+\eta(r, \phi, t)$, where $b$ is the inner radius of the fluid free surface for an undisturbed motion, $\eta$ is the response function of the fluid free surface to the disturbance. For small perturbations, $R_{b} \approx b$, the boundary conditions at the fluid free surface are

$$
p(b)=0, \quad u(b)=\frac{\partial \eta}{\partial t}
$$

For the viscous fluid, the velocity in the $\phi$ direction must be zero at the wetted rotor surface (no slip condition) and the free surface of the fluid must be free from shear stresses, i.e.,

$$
\begin{gathered}
v(a)=0 \\
\left.\sigma_{r \phi}\right|_{r=b}=\left.\frac{1}{b} \frac{\partial u}{\partial \phi}\right|_{r=b}+\left.\frac{\partial v}{\partial r}\right|_{r=b}-\left.\frac{v}{b}\right|_{r=b}=0
\end{gathered}
$$

In order to linearize Eqs. (1)-(3), a perturbation method is employed in which the eccentricity ratio of the chamber motion relative to the inner radius of the chamber $\varepsilon$ is chosen as the perturbation parameter. Each dependent variable, $\Phi \in[u, v, p]$, is represented in powers of the non-dimensional eccentricity ratio $\varepsilon$, i.e., $\Phi=\Phi_{0}+\varepsilon \Phi_{1}+O\left(\varepsilon^{2}\right)$, where $\Phi_{0}$ is the zero-order term and $\Phi_{1}$ the first-order term. After substituting the relation in Eqs. (1)-(3) and neglecting the higher-order terms of $\varepsilon$, the terms of like order are grouped to form the corresponding equations for different order solutions.

Because the zero-order solution corresponding to the undisturbed motion of the rotating fluid can be considered as the rigid body motion which rotates at the same rotational speed of the chamber, so the zero-order solution is given by:

$$
u_{0}=0, \quad v_{0}=0, \quad p_{0}=\frac{1}{2} \rho \Omega^{2}\left(r^{2}-b^{2}\right)
$$

On the basis of the zero-order solution, we get the equations to be satisfied by the first-order solution, which can be expressed in non-dimensional parameters as

$$
\frac{\partial\left(R U_{1}\right)}{\partial R}+\frac{\partial V_{1}}{\partial \phi}=0
$$

$$
\begin{aligned}
\frac{\partial U_{1}}{\partial \tau}-2 V_{1}= & -\frac{1}{2} \frac{\partial P_{1}}{\partial R} \\
& +\frac{1}{R e}\left(\frac{\partial^{2} U_{1}}{\partial R^{2}}+\frac{1}{R} \frac{\partial U_{1}}{\partial R}+\frac{1}{R^{2}} \frac{\partial^{2} U_{1}}{\partial \phi^{2}}\right. \\
& \left.\quad-\frac{U_{1}}{R}-\frac{2}{R^{2}} \frac{\partial V_{1}}{\partial \phi}\right) \\
& +\alpha^{2} \exp [i(\sigma \tau-\phi)] \\
\frac{\partial V_{1}}{\partial \tau}+2 U_{1}= & -\frac{1}{R} \frac{\partial P_{1}}{\partial \phi} \quad-\frac{1}{R e}\left(\frac{\partial^{2} V_{1}}{\partial R^{2}}+\frac{1}{R} \frac{\partial V_{1}}{\partial R}+\frac{1}{R^{2}} \frac{\partial^{2} V_{1}}{\partial \phi^{2}}\right. \\
& \left.-\frac{V_{1}}{R}+\frac{2}{r^{2}} \frac{\partial U_{1}}{\partial \phi}\right) \\
& -\alpha^{2} i \exp [i(\sigma \tau-\phi)]
\end{aligned}
$$

The boundary conditions are

$$
\begin{gathered}
U_{1}(1)=0 \\
V_{1}(1)=0 \\
P_{1}(H)=-2 H \varsigma_{1} \\
U(H)=\frac{\partial \varsigma_{1}}{\partial \tau} \\
\left.\frac{1}{H} \frac{\partial U_{1}}{\partial \phi}\right|_{R=H}+\left.\frac{\partial V_{1}}{\partial R}\right|_{R=H}-\left.\frac{V_{1}}{H}\right|_{R=H}=0
\end{gathered}
$$

where $U_{1}=\left(u_{1} / a \Omega\right), \quad V_{1}=\left(v_{1} / a \Omega\right), \quad \operatorname{Re}=\left(a^{2} \Omega / \mu\right)$, $P_{1}=\left(2 p_{1} / a^{2} \rho \Omega^{2}\right), \quad R=(r / a), \quad H=(b / a), \alpha=(\omega / \Omega)$, $\varsigma_{1}=\left(\eta_{1} / a\right), \tau=\Omega t, \sigma=\alpha-1$.

It is found that the forced solution of Eqs. (8)-(10), in which we are interested, should be in the form of $\Psi_{1}=\bar{\Psi}(R) \cdot \exp [i(\sigma \tau-\phi)]$, 
$\Psi_{1} \in\left[U_{1}, V_{1}, P_{1}, \varsigma_{1}\right]$, so we get from Eqs. (8)-(10)

$$
\begin{gathered}
\bar{V}=-i \bar{U}-i R \frac{d \bar{U}}{d R} \\
\bar{P}=-2 i(\alpha-1) R^{2} \frac{d \bar{U}}{d R}-2(\alpha+1) R i \bar{U}+2 \alpha^{2} R \\
+\frac{2 R}{R e}\left(R \frac{d^{3} \bar{U}}{d R^{3}}+4 \frac{d^{2} \bar{U}}{d R^{2}}\right) \\
{\left[\left(\frac{d^{2}}{d R^{2}}+\frac{1}{R} \frac{d}{d R}-\frac{1}{R^{2}}\right)-(\alpha-1) R e i\right]} \\
\left(R \frac{d^{2}}{d R^{2}}+3 \frac{d}{d R}\right) \bar{U}=0 .
\end{gathered}
$$

The corresponding boundary conditions for variable $\bar{U}$ are

$$
\begin{gathered}
\bar{U}(1)=0 \\
\left.\frac{d \bar{U}}{d R}\right|_{R=1}=0 \\
\left.H \frac{d^{2} \bar{U}}{d R^{2}}\right|_{R=H}+\left.\frac{d \bar{U}}{d R}\right|_{R=H}=0
\end{gathered}
$$

After eliminating the unknown variable $\varsigma_{1}$ from the Eqs. (13)-(14) and using Eq. (16), we can convert the boundary conditions in Eqs. (13)-(14) into the following form.

$$
\begin{aligned}
& \left.(\alpha-1) H^{2} \frac{d^{3} \bar{U}}{d R^{3}}\right|_{R=H}+\left.4(\alpha-1) H \frac{d^{2} \bar{U}}{d R^{2}}\right|_{R=H} \\
& -\left.(\alpha-1)^{2} \operatorname{ReH}^{2} i \frac{d \bar{U}}{d R}\right|_{R=H}-\left.\alpha^{2} \operatorname{ReHi} \bar{U}\right|_{R=H} \\
& +\alpha^{2}(\alpha-1) \operatorname{ReH}=0
\end{aligned}
$$

Equation (18) can be converted into a first-order modified Bessel's equation, the solution of $\bar{U}$ can be written as:

$$
\bar{U}=C_{1}+C_{2} \frac{1}{R^{2}}+\frac{C_{3}}{R} I_{1}(k R)+\frac{C_{4}}{R} K_{1}(k R)
$$

$C_{i}(i=1,2,3$ and 4$)$ are integral constants which are determined by the boundary conditions Eqs.
(19) - (22). $I_{1}(k R)$ and $K_{1}(k R)$ are modified Bessel's functions of the first order first kind and the second order first kind, respectively. $k=(1 / 2)$ $[1+\operatorname{sig}(\alpha-1) i] \sqrt{|\alpha-1| R e}$. After obtaining $\bar{U}$, we can easily get $\bar{V}$ and $\bar{P}$ from Eqs. (16) and (17), respectively.

Since the influence of the fluid viscosity is considered here, the net fluid forces acting on the rotor depend on both the fluid pressure and the shear stress at the wetted rotor surface, and are obtained by integrating them in a certain range. Because $p(a)$ and $\sigma_{r \phi}(a)$ obtained are all complex values, only the real parts of them are physically of sense, so the fluid forces acting on the rotor are

$$
\begin{aligned}
& \left\{\begin{array}{l}
F_{X} \\
F_{Y}
\end{array}\right\}=a L \\
& \int_{0}^{2 \pi}\left\{\begin{array}{l}
\operatorname{Real}[p(a)] \cos \phi+\operatorname{Real}\left[\sigma_{r \phi}(a)\right] \sin \phi \\
\operatorname{Real}[p(a)] \sin \phi-\operatorname{Real}\left[\sigma_{r \phi}(a)\right] \cos \phi
\end{array}\right\} d \phi .
\end{aligned}
$$

Finally, we get the net forces $F_{X}$ and $F_{Y}$ in the $X$ and $Y$ directions in the fixed co-ordinate system as follows:

$$
\left\{\begin{array}{l}
F_{X} \\
F_{Y}
\end{array}\right\}=-\left[\begin{array}{cc}
-\operatorname{Real}(A) & -\operatorname{Real}(A i) \\
\operatorname{Real}(A i) & -\operatorname{Real}(A)
\end{array}\right]\left\{\begin{array}{c}
X \\
Y
\end{array}\right\}
$$

where

$$
A=\pi \rho a^{2} L \Omega^{2}\left\{\alpha^{2}+\frac{1}{R e}\left[\left.\frac{d^{3} \bar{U}}{d R^{3}}\right|_{R=1}+\left.3 \frac{d^{2} \bar{U}}{d R^{2}}\right|_{R=1}\right]\right\}
$$

$L$ is the height of the chamber.

We should point out, the linearized forces of the rotating fluid layer acting on the rotor only depend on the displacement of chamber center, other parameters of the chamber structure and properties of the fluid, do not depend on velocity of the chamber center. It is distinctly different from the traditional unstable elements in rotor systems, where a destabilizing force would typically be tangential with respect to whirl orbit 
and contain a velocity proportional and a deflection proportional term, i.e., there exist both the damping and the stiffness effect. However, There exists only the stiffness effect not any damping effect between the rotating fluid layer and the rotor even for the viscous fluid model. This should be studied in the rotating viscous fluid dynamics in the future.

\section{ROTOR SYSTEM MODEL}

The rotor system may be one of the systems shown in Figure 1. One consists of a disk with a cylindrical chamber mounted midway between the bearings on a massless uniform elastic shaft used by Wolf (1968). The other consists of a hollow rigid rotor partially filled with fluid mounted symmetrically on the flexible supports. If only the motion of parallel mode is considered, these two rotor systems can be simplified as a signal mass-stiffness-damper system. Let the equivalent mass of the rotor system be $M$, the equivalent external damping be $C$ and the equivalent stiffness coefficient of the system be $K$, respectively, the equations of motion of the rotor system in fixed $X Y Z$ co-ordinate system are given by

$$
\begin{aligned}
{\left[\begin{array}{cc}
M & 0 \\
0 & M
\end{array}\right]\left\{\begin{array}{l}
\ddot{X} \\
\ddot{Y}
\end{array}\right\} } & +\left[\begin{array}{cc}
C & 0 \\
0 & C
\end{array}\right]\left\{\begin{array}{l}
\dot{X} \\
\dot{Y}
\end{array}\right\} \\
& +\left[\begin{array}{cc}
K & 0 \\
0 & K
\end{array}\right]\left\{\begin{array}{l}
X \\
Y
\end{array}\right\}=\left\{\begin{array}{l}
F_{X} \\
F_{Y}
\end{array}\right\}
\end{aligned}
$$

Assuming the solution of Eq. (26) to have the form of $\Theta=\bar{\Theta} \exp (i \lambda t), \Theta \in[X, Y]$, we get a homogeneous system of equations to which nontrivial solutions only exist if the determinant equals zero. After some algebraic operation, we obtain the following characteristic equation of the rotor system partially filled with viscous fluid:

$$
\begin{aligned}
\bar{\lambda}^{4} S^{4}- & 2 \xi \bar{\lambda}^{3} S^{3}-\left[2+\xi^{2}-2 \operatorname{Real}(\bar{A})\right] \bar{\lambda}^{2} S^{2} \\
+ & 2[1-\operatorname{Real}(\bar{A})] \bar{\lambda} i \xi S \\
+ & {\left[(1-\operatorname{Real}(\bar{A}))^{2}+(\operatorname{Real}(\bar{A} i))^{2}\right]=0 }
\end{aligned}
$$

where

$$
\bar{A}=\bar{m} S^{2}\left\{\alpha^{2}+\frac{1}{S R e_{\mathrm{cr}}}\left[\left.\frac{d^{3} \bar{U}}{d R^{3}}\right|_{R=1}+\left.3 \frac{d^{2} \bar{U}}{d R^{2}}\right|_{R=1}\right]\right\}
$$

$\alpha=\operatorname{Real}(\bar{\lambda}) \cdot \xi=C / M \omega_{\text {cr }}$ is the external damping ratio, $\bar{\lambda}=\lambda / \Omega$ is the non-dimensional complex

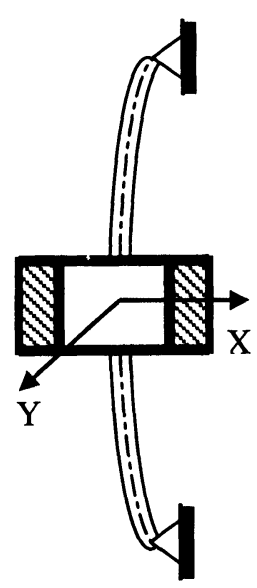

a

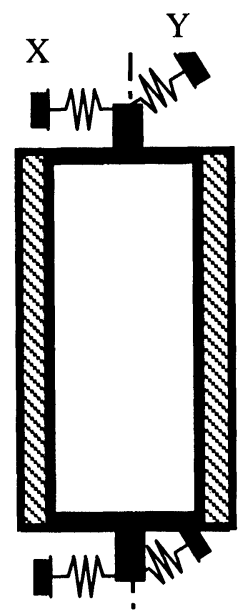

b

FIGURE 1 Rotor system models. 
eigen-value, $\bar{m}=m_{F} / M$ is the mass ratio, $S=$ $\Omega / \omega_{\mathrm{cr}}$ is the rotational speed ratio, $m_{F}=\pi \rho L a^{2}$ is the mass of the fluid needed to completely fill the chamber, $\omega_{\mathrm{cr}}^{2}=K / M$ is the first critical speed of the empty rotor system.

It is clear that the instability behavior of the rotor partially filled with the viscous incompressible fluid will be described by five non-dimensional parameters $H, S, R e_{\mathrm{cr}}, \bar{m}$ and $\xi$. The stability analysis is performed by looking at the smallest imaginary part $\operatorname{Im}(\bar{\lambda})_{\min }$ occurring among the four eigen-values in Eq. (27) for each rotational speed ratio $S$. If $\operatorname{Im}(\bar{\lambda})_{\min }$ is less than zero, the rotor system is unstable; If $\operatorname{Im}(\bar{\lambda})_{\min }$ is larger than zero, the rotor system is stable. Since the eigen-value $\bar{\lambda}$ to be obtained appears in the arguments of the Bessel's functions, the iteration method must be used in order to solve Eq. (27) for $\bar{\lambda}$.

\section{RESULTS AND DISCUSSION}

The following analyses are performed for rotational speed ratios $0.01<S<2.0$, Reynolds number $10<R e_{\text {cr }}<10^{5}$, fluid fill ratio $0.01<H<1.0$, external damping ratio $0.0005<\xi<0.2$ and mass ratio $0.001<\bar{m}<1.0$. It is found that there exist two forms in curve of the smallest imaginary part $\operatorname{Im}(\bar{\lambda})_{\min }$ among the eigen-values varying with the rotational speed ratio $S$ shown in Figure 2. One shown in dashed line just has one top in which the $\operatorname{Im}(\bar{\lambda})_{\min }$ is more than zero, there is only one unstable region of rotational speeds; the other shown in solid line has two tops, so there exist two unstable regions of rotational speeds. The reason for the occurrence of two unstable regions of rotational speeds in the isotropic rotors is that the linearized fluid forces acting on the rotor are not isotropic.

\section{Effect of the Reynolds Number, $R e_{\text {cr }}$}

The influence of the Reynolds number at the critical speed $R e_{\text {cr }}$ on the unstable regions is shown in Figure 3 for different fluid fill ratios $H$ 's. It shows that when $R e_{\mathrm{cr}}$ is small, the two unstable

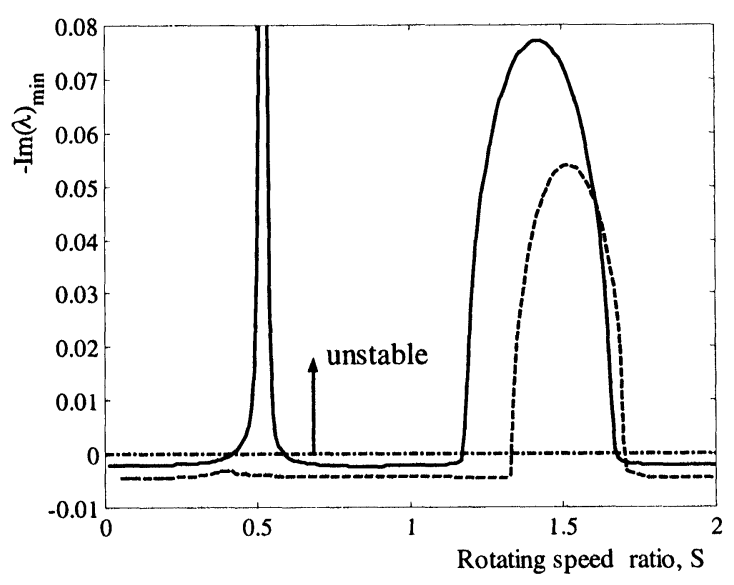

FIGURE 2 The smallest imaginary part $\operatorname{Im}(\bar{\lambda})_{\min }$ varying with the rotating speed ratio $S$.

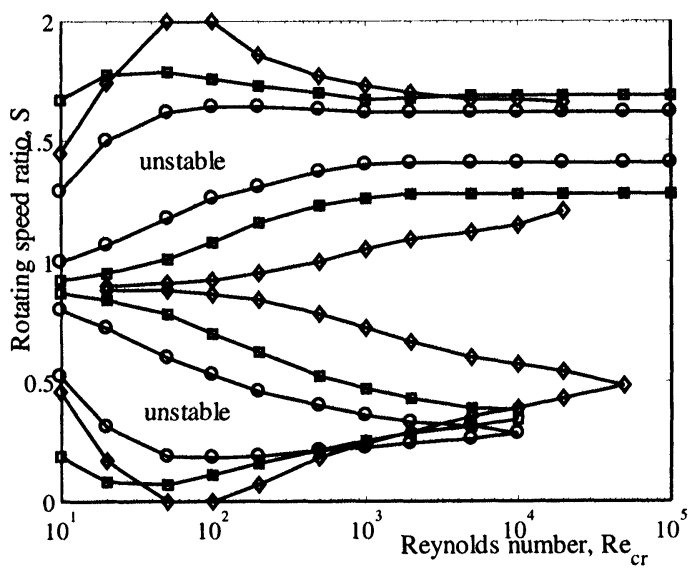

FIGURE 3 Effect of Reynolds number $R e_{\mathrm{cr}}$ on rotor instability. $\quad \xi=0.01, \quad \bar{m}=0.25, \quad H=0.25(\circ), \quad H=0.50(\square)$, $H=0.75(\diamond)$.

regions may appear, one is above the critical speed and the other under the critical speed. As $R e_{\mathrm{cr}}$ increases, the two unstable regions first expand, then narrow, especially the lower one. If $R e_{\mathrm{cr}}$ is higher, the lower unstable region disappears and there exists only the upper one. This may be the reason why only one unstable region is observed in experiments with higher Reynolds number where water with very low viscosity is often used as the working fluid. The larger $H$, the wider the unstable regions become. However, for small and medium $H$, the change of the instability boundaries of the 
upper unstable region with $R e_{\mathrm{cr}}$ in the higher $R e_{\mathrm{cr}}$ region is very little. It means the fluid viscosity is not the key factor to result in the rotor instability. Even if the fluid viscosity is very small, the rotor instability can also appear.

\section{Effect of the Fluid Fill Ratio, $H$}

The fluid fill ratio $H$ is the ratio of the inner radius of the undisturbed rotating fluid layer to the inner radius of the chamber. The variation of the instability regions with $H$ is given in Figure 4 for different external damping ratios $\xi$ 's. It shows that when $H$ is very small or very large, the rotor system is absolutely stable, i.e., no unstable motion appears in the speed range considered. If $R e_{\mathrm{cr}}$ is high, there exists only one unstable region above the critical speed of the empty rotor system, but there probably exist two unstable regions for the lower $R e_{\mathrm{cr}}$. As $H$ increases, the unstable motions will appear in the two regions of rotational speeds, the lower boundaries of these unstable regions decrease and the upper ones increase, two unstable regions expand rapidly. If $\xi$ is small, for example $\xi=0.005$ and $0.6<H<0.9$, the unstable regions expand to almost the whole rotational speed range considered except a very small zone between two unstable regions. This means the rotor system will

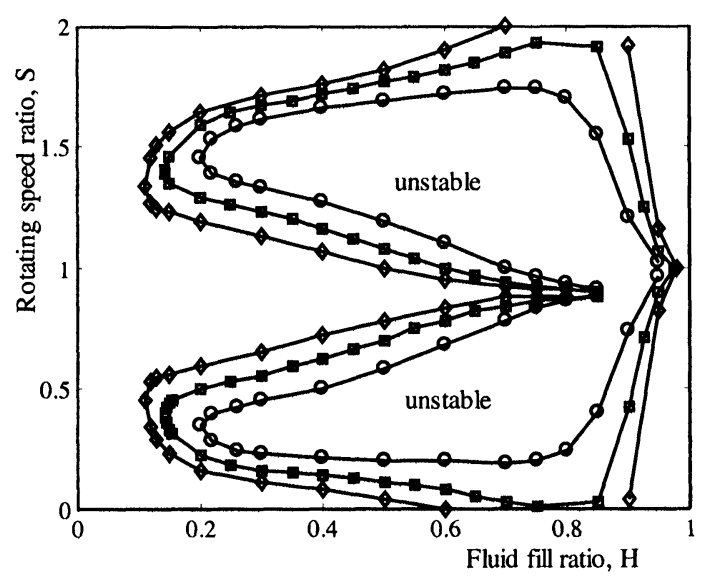

FIGURE 4 Effect of fluid fill ratio $H$ on rotor instability. $R e_{\mathrm{cr}}=10^{2}, \bar{m}=0.25, \xi=0.025(\circ), \xi=0.01(\square), \xi=0.005(\diamond)$. be unstable in very low rotational speeds. As $H$ further increases into the region of very high, the two unstable regions become narrow, then change to one unstable region and finally disappear. The smaller $\xi$, the wider the unstable regions will be, and the wider the range of $H$ for occurring unstable motions is. Similar phenomenon in which the rotor system will become absolutely stable in either the very large $H$ or very small $H$ was also obtained by Saito and Someya (1980) for the thin fluid layer, but they did not find there also exists an absolutely stable phenomenon in the case of very small $H$.

\section{Effect of the External Damping Ratio, $\xi$}

Figure 5 gives the result of the influence of external damping ratio $\xi$ on the unstable regions at different $R e_{\mathrm{cr}}$ 's and $H$ 's. When $H$ is very large in Figure 5a, i.e., the fluid layer in the chamber is very thin, there is only one unstable region. An increase in $\xi$ tends to make the rotor system more stable by rapidly narrowing the unstable region. When $\xi$ is over a certain value, the system will be absolutely stable. This result is in good agreement with the theoretical results obtained by Saito and Someya (1980) with the thin fluid layer theory and the experimental observation of Kaneko and

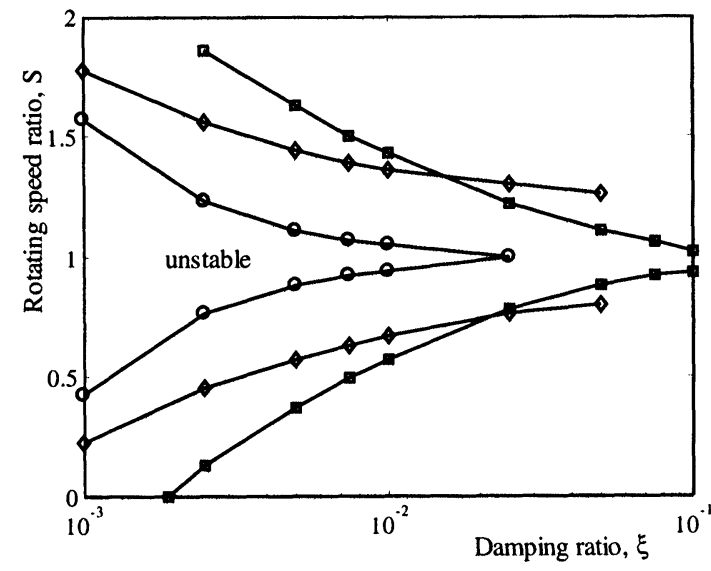

FIGURE 5a Effect of external damping ratio $\xi$ on rotor instability. $H=0.98, \bar{m}=0.15, R e_{\mathrm{cr}}=10^{3}(\circ), \quad R e_{\mathrm{cr}}=10^{4}(\square)$, $R e_{\mathrm{cr}}=10^{5}(\diamond)$ 


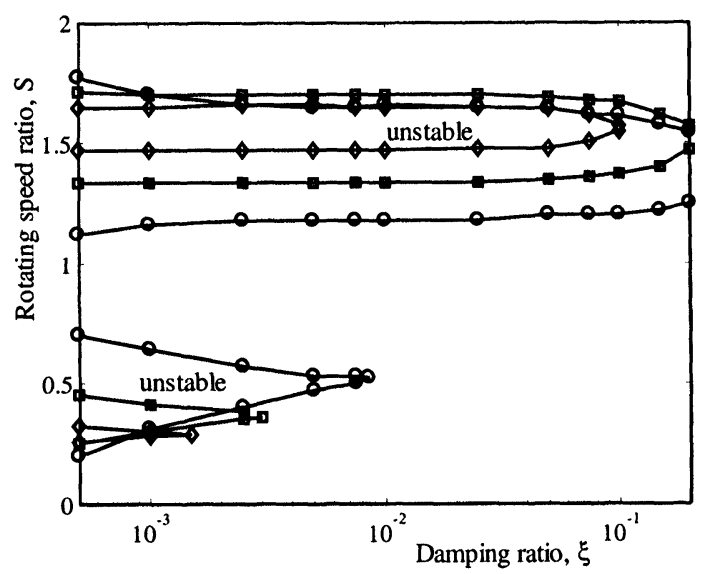

FIGURE 5b Effect of external damping ratio $\xi$ on rotor instability. $R e_{\mathrm{cr}}=10^{5}, \bar{m}=0.175, H=0.75(\circ), H=0.50(\square)$, $H=0.25(\diamond)$

Hayama (1985) and Ota et al. (1986), but contrary to Hendricks and Morton's results (1979). The probable reason for this difference is that different variables are used as perturbation parameter in linearizing the motion's equation of the rotating fluid and they respectively analyzed the different regions of instability of the rotor system.

If $R e_{\mathrm{cr}}$ is higher, for example $R e_{\mathrm{cr}}=10^{5}$ in Figure $5 \mathrm{~b}$, there are two unstable regions when $\xi$ is much smaller. Two unstable regions will narrow with the increase of $\xi$, especially the lower one. When $\xi$ is over a certain value, the lower unstable region disappears and there is only the upper one above the critical speed of the empty rotor system. However, for very high $R e_{\mathrm{cr}}$, the influence of $\xi$ on the upper unstable region is not obvious in a certain range of $\xi$, but it does not expand the unstable region. If $\xi$ is large enough, the system could also be stabilized, which agrees well with the experimental observation obtained by Kaneko and Hayama (1985).

\section{Effect of the Mass Ratio, $\bar{m}$}

The mass ratio $\bar{m}$ is the ratio of fluid mass completely filling the chamber to rotor mass. Figure 6 gives the instability regions varying with $\bar{m}$ under different $H$ 's in case of lower $R e_{\text {cr. }}$. When

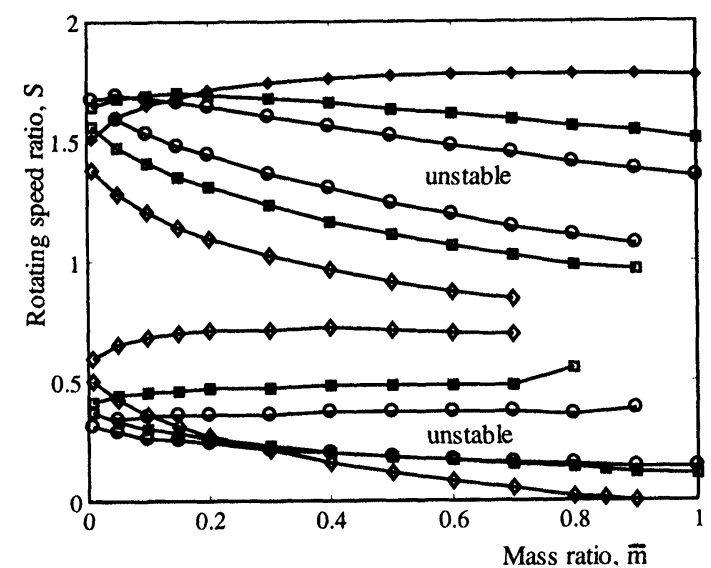

FIGURE 6 Effect of mass ratio $\bar{m}$ on rotor instability. $R e_{\mathrm{cr}}=10^{3}, \xi=0.01, H=0.25(\circ), H=0.50(\square), H=0.75(\diamond)$.

$H$ is larger, as $\bar{m}$ increases, the lower instability boundaries decrease, the upper ones increase, the unstable regions expand. If $H$ is not large, as $\bar{m}$ increases, both the lower and upper instability boundaries decrease, but the unstable regions expand. Therefore, the unstable regions always expand with the increase of $\bar{m}$. When $\bar{m}$ is larger, the lower instability boundary of the upper unstable region may be below the critical speed of the empty rotor system.

\section{CONCLUSIONS}

On the basis of the linearized fluid forces in the 2D-case obtained directly by using the NavierStokes equations, this paper investigates the stability of symmetrical rotors partially filled with viscous incompressible fluid and analyses the effects of the parameters of the rotor system on the stability regions. It is shown that for isotropic rotors, the viscous fluid model may predict two unstable regions, one is generally above the critical speed of the empty rotor system and the other is below the critical speed. The lower instability boundary of the upper unstable region may also be less than the critical speed in some cases.

In the viscous fluid model point of view, adding external damping makes the system more stable 
and may be an effective method to suppress the unstable motion in certain cases.

The influence of the rotor system parameters on the instability regions are more complex, it is difficult to summarize some general rules. For different regions of the system parameters and their combinations, the conclusions may be different.

It is also shown that there are many other problems to be studied. For example, does there exist any damping effect between the rotating fluid layer and the rotor? Are there two unstable regions in the lower Reynolds number? Why are the results about the effect of external damping on the unstable regions by just using different linearizing methods on the Navier-Stokes equations different? In order to check the correctness of the modeling and simplification, it is very necessary to perform systematic experiments on the instability of rotor partially filled with fluid.

\section{Acknowledgements}

The supports of NNSF of China, the Alexander von Humboldt Foundation of Germany and SRF for ROCS, SEM are gratefully acknowledged. The Author thanks Prof. H. Ulbrich of Essen University, Germany, for his kind help during his stay in Germany.

\section{NOMENCLATURE}

a inner radius of the chamber

$A, \bar{A} \quad$ variables defined in this paper

$b$ inner radius of undisturbed fluid free surface

C equivalent external damping coefficient of rotor system

$C_{i} \quad$ integral constant

$F_{x}, F_{y}$ fluid force components in the $x$ and $y$ directions

$H \quad$ fluid fill ratio, $H=b / a$

$i \quad$ complex unit, $i=\sqrt{-1}$

$I_{1}$ modified Bessel's function of first kind first order $k \quad$ variable defined in this paper

$K$ equivalent stiffness coefficient of rotor system

$K_{1} \quad$ modified Bessel's function of second kind first order

$L \quad$ length of the chamber

$m_{F} \quad$ mass of the fluid need to completely fill the chamber, $m_{F}=\pi \rho L a^{2}$

$\bar{m} \quad$ mass ratio, $\bar{m}=m_{F} / M$

$M \quad$ equivalent mass of empty rotor

$p \quad$ fluid pressure

$\bar{P} \quad$ non-dimensional fluid pressure

$r, \phi$ polar co-ordinates in the rotating coordinate system

$R$ non-dimensional radius, $R=r / a$

$R e \quad$ Reynolds number at the rotational speed of $\Omega, R e=\left(a^{2} \Omega / \mu\right)=S R e_{\mathrm{cr}}$

$R e_{\mathrm{cr}} \quad$ Reynolds number at the critical speed $\omega_{\mathrm{cr}}$, $R e_{\mathrm{cr}}=\left(a^{2} \omega_{\mathrm{cr}} / \mu\right)$

$S$ rotating speed ratio, $S=\Omega / \omega_{\mathrm{cr}}$

$t \quad$ time

$u, v \quad$ velocity components in the $r$ - $\phi$ co-ordinate system

$U, V$ non-dimensional velocity components

$X, Y$ co-ordinates of the chamber mass centre

$\alpha$ non-dimensional whirling speed ratio, $\alpha=\omega / \Omega$

$\varepsilon \quad$ eccentricity ratio of the chamber center relative to inner radius of the chamber $a$

$\eta \quad$ response function of free surface of the fluid to the disturbance

$\zeta_{1}$ non-dimensional response function of free surface to the disturbance, $\zeta_{1}=\left(\eta_{1} / a\right)$

$\lambda \quad$ complex eigen-value

$\bar{\lambda}$ non-dimensional complex eigen-value, $\bar{\lambda}=(\lambda / \Omega)$

$\sigma_{r \phi} \quad$ shear stress

$\tau \quad$ non-dimensional time, $\tau=\Omega t$

$\mu \quad$ fluid kinematic viscosity

$\rho \quad$ fluid density

$\xi \quad$ external damping ratio, $\xi=C / M \omega_{\mathrm{cr}}$

$\omega \quad$ whirling speed of the rotor system

$\omega_{\mathrm{cr}} \quad$ first critical speed of empty rotor system, $\omega_{\mathrm{cr}}^{2}=(K / M)$

$\Delta \quad$ Laplacian operator

$\Omega \quad$ rotational speed 


\section{Subscripts}

$0 \quad$ zero-order term

1 first-order term

\section{References}

Hendricks, S. L. and Morton, J. B. (1979) Stability of a Rotor Partially Filled with a Viscous Incompressible Fluid, ASME J. of Applied Mechanics, 46(4), 913-918.

Holm-Christensen, O. and Träger, K. (1991) A Note on Rotor Instability Caused by Liquid Motions, ASME J. of Applied Mechanics, 58(3), 804-811.

Kaneko, S. and Hayama, S. (1985) Self-excited Oscillation of a Hollow Rotating Shaft Partially Filled with a Liquid (1st
Report, Instability Based on the Fluid Force Obtained from Boundary Layer Theory), Bulletin of JSME, 28(246), 2994-3001.

Kollmann, F. G. (1962) Experimentelle und theoretische Untersuchungen über die Kritischen Drehzahlen flüssigkeitsgefulter Hohlkörper, Forschund auf dem Gebiete des Ingenieurweasns, Ausgabe $B, \quad 28,115-123$ and $147-153$.

Ota, H., Ishida, Y., Sato, A. and Yamada, T. (1986) Experiments on Vibrations of a Hollow Rotor Partially Filled with Fluid, Bulletin of JSME, 29(256), 3520-3529.

Saito, S. and Someya, T. (1980) Self-Excited Vibration of a Rotating Hollow Shaft Partially Filled with Liquid, ASME J. of Mechanical Design, 102(1), 185-192.

Wolf, J. A. Jr. (1968) Whirl Dynamics of a Rotor Partially Filled with Liquid, ASME J. of Applied Mechanics, 35(4), 676-682. 

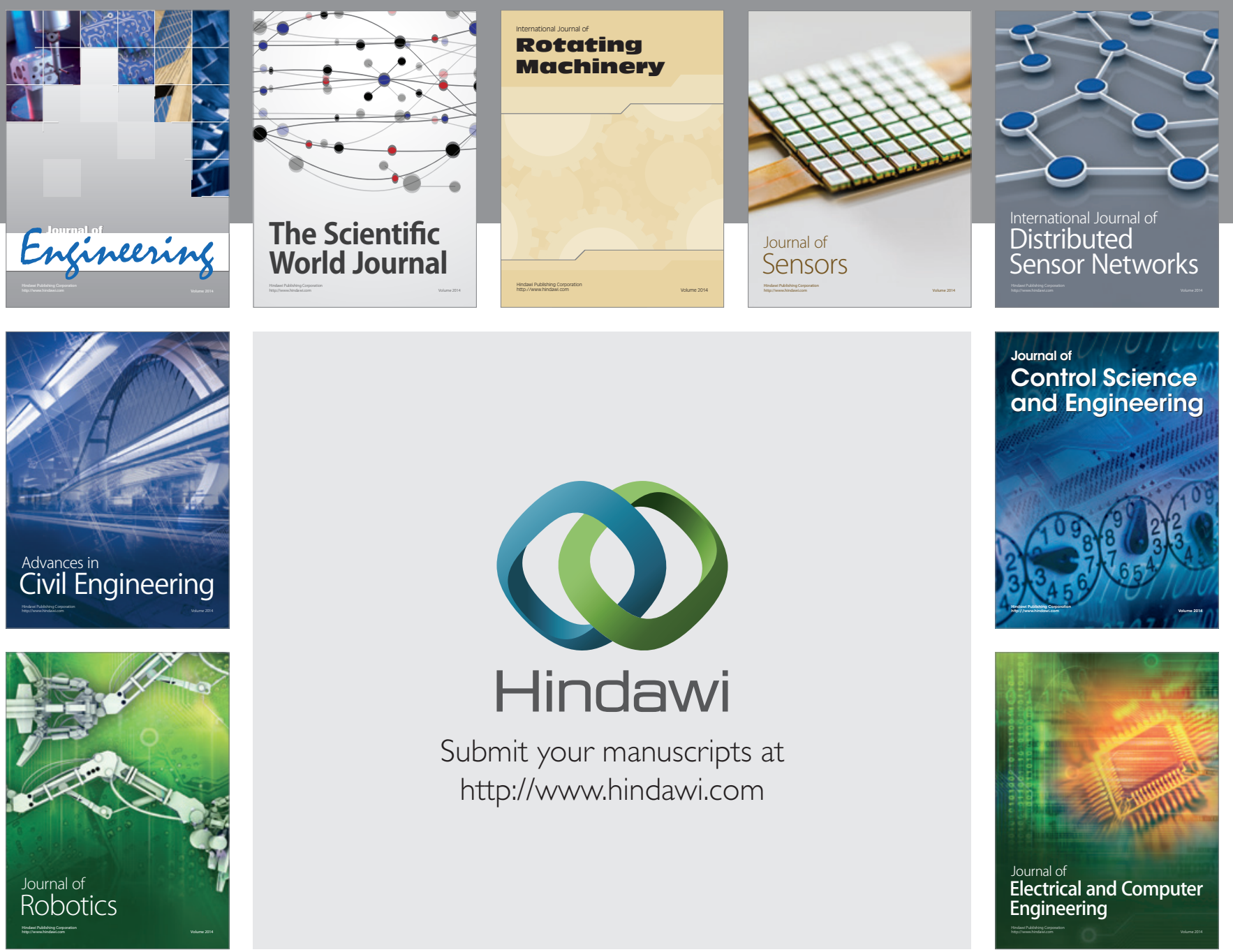

Submit your manuscripts at

http://www.hindawi.com
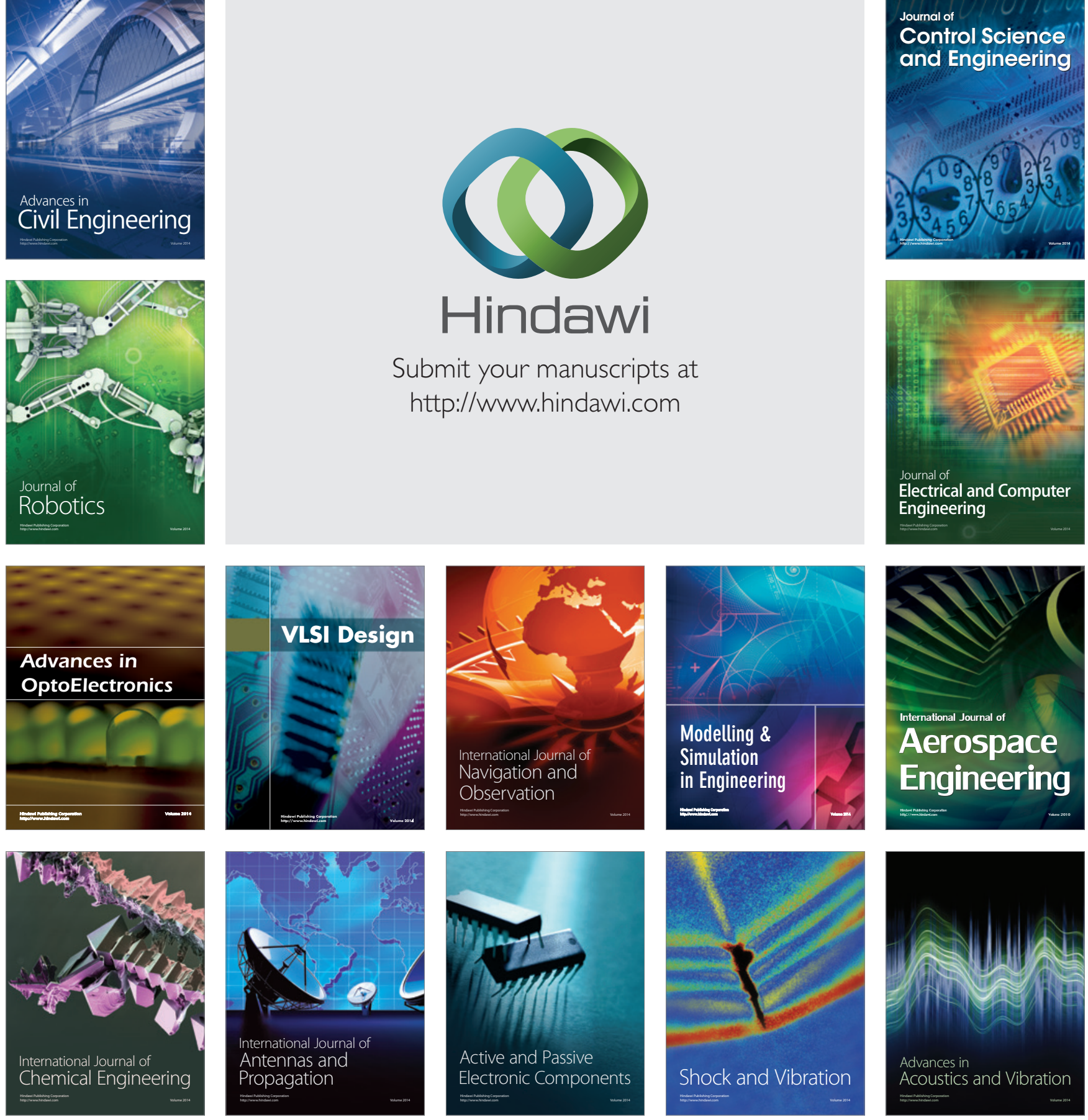